\title{
Assessing the Viability of a Vocal Cord Vibration Switch for Four Children with Multiple Disabilities
}

\author{
Elaine C. Lu ${ }^{1,2}$, Tiago H. Falk ${ }^{*}, 1,2$, Gail Teachman ${ }^{1}$ and Tom Chau ${ }^{1,2}$

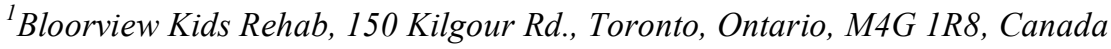 \\ ${ }^{2}$ Institute of Biomaterials and Biomedical Engineering, University of Toronto, Rosebrugh Building, 164 College St., Rm. \\ 407, Toronto, Ontario, M5S 3G9, Canada
}

\begin{abstract}
This prospective case series evaluated the viability of a recently-developed vocal cord vibration switch with four participants between the ages of 5.8-14.5 years old who had severe motor disabilities and complex communication needs. At the time of study enrolment, participants did not have an established single switch access pathway but were all capable of some form of vocalization, albeit frequently unintelligible. Participants were each provided with a vocal cord vibration switch for unrestricted home use. Participants were subsequently followed for a one-month period and switch viability was evaluated on three separate occasions. Viability of the switch was assessed in terms of quantitative measures such as switch sensitivity, specificity, and user-perceived exertion, along with qualitative characterizations such as ease of use and user satisfaction reported by the participants, their caregivers, and their clinicians. Data from the three consecutive assessments indicated low levels of exertion, device sensitivity between $73.3 \%$ and $93.2 \%$, and specificity between $98.1 \%$ and $100 \%$. All four participants reported that they liked the device and expressed desire to continue using it. In addition, clinicians and caregivers unanimously agreed that the device was the most viable access solution for their clients.
\end{abstract}

Keywords: Augmentative and alternative communication (AAC), vocal cord vibration, access switch, severe disabilities, complex communication needs.

\section{INTRODUCTION}

The need to communicate is innate to every human being. From a child's first cry to a person's final words, the desire to communicate is implicit. For individuals with severe and multiple disabilities, however, this desire is often met with frustration as communication can be hindered and desires are not readily understood [1]. As an example, worldwide approximately one in one hundred individuals have complex communications needs and cannot rely on speech for daily communication [2]. It is with this in mind that augmentative and alternative communication (AAC) strategies have been developed.

AAC is a process, a set of strategies and approaches that supplements or replaces natural speech or writing for those who are unable to meet their communication needs through natural methods [3]. Many who have complex communication needs are able to express themselves, but do not have a way to be understood. A 2006 report of Canadian census data found that $71.9 \%$ of adults and $51.5 \%$ of children with communication disabilities had none of their communication needs met with regards to assistive devices [4]. It is known that lack of communication not only affects social participation, education, and employment [5], but may also profoundly limit cognitive and social development in children with disabilities [6]. Access technologies can play a

\footnotetext{
*Address correspondence to this author at the Institute of Biomaterials and Biomedical Engineering, University of Toronto, Rosebrugh Building, 164 College St., Rm. 407, Toronto, Ontario, M5S 3G9, Canada; Tel: 01-416425-6220, Ext. 3326; Fax: 01-416-425-1634;

E-mail: tiago.falk@ieee.org
}

key role in fostering communications and in enabling interaction with the surrounding environment. Traditionally, mechanical switches have served as an interface between the users and their computers, environmental controls, or communication aids. For individuals with severe motor disabilities, however, mechanical switch operation may be difficult, if not impossible [7].

Amongst the population of individuals with difficulties accessing AAC technologies are those who are able to vocalize. However, due to dysarthria, poor respiratory control, or laryngeal dysfunction, produced vocalizations may not be comprehensible to the general population, or consistent enough for speech recognition systems [8]. To this end, microphone-based voice activated switches have been used and proven to be effective $[9,10]$. Such solutions, however, suffer from problems associated with low-volume sounds, unintended sounds (such as coughing), and environmental noise [8]. To overcome some of these issues, throat microphones, in combination with conventional airborne microphones, have been explored [11]. Combined microphone setups, however, are still susceptible to false switch activations that occur due to coughs, throat clearings, heavy breathing, congested airways, or involuntary spastic head movements [11]. Such limitations can be overcome with the use of a recently-proposed periodic vocal cord vibration detection switch [12] described in more detail in section 2.2.

This prospective case series investigated the viability of the vocal cord vibration detection switch as an access technology for four children with severe motor disabilities and complex communication needs. In this study, switch 
viability was assessed in terms of quantitative measures such as switch sensitivity and specificity, user-perceived exertion, as well as qualitative characterizations of switch usage such as ease of use, and user satisfaction reported by the participants and their caregivers.

\section{METHOD}

\subsection{Participant Descriptions}

Four children and youth between the ages of 5.8 and 14.5 (mean 10.3 years) with multiple disabilities and complex communication needs were identified as needing an access solution by occupational therapists working in a communication and writing aids service at a children's rehabilitation center. These participants were chosen because they were able to vocalize voluntarily. Despite having tried several commercially-available switches, none of the participants possessed a reliable access method. Participants, their caregivers, and their therapists were recruited to be a part of this study and informed consent was obtained from the parents. The four participants are described in more detail in the subsections to follow; pseudonyms are used.

\subsubsection{Participant 1: Sam}

Sam was a 5.8 year old male, who was diagnosed with ataxic spastic quadriplegic cerebral palsy. He had bilateral hearing loss and wore hearing aids and used glasses to correct for myopia. He used a wheelchair and had limited controlled movement of his head, arms, legs, and trunk. Cognitively, he was considered to be functioning at an ageappropriate level and was able to understand speech as well as speak, although softly and sometimes unintelligibly. His breathing was raspy at times. He had a history of using a 2.5 inch button-style mechanical switch positioned at various locations - near his foot, behind his elbow, or near his chin. Usage of this switch, however, was overly fatiguing and was discontinued. Sam was interested in a reliable switch alternative for playing games and listening to stories on a computer. His occupational therapist had worked with him for one year.

\subsubsection{Participant 2: John}

John was a 9.0 year old male. He was diagnosed with quadriplegic dystonic cerebral palsy. As a result of bilateral hearing loss he wore hearing aids but had good eyesight. John used a wheelchair and had extremely limited voluntary control over his neck, head, legs and arms. When his left arm was stabilized he had some control of the fingers of his left hand. He was reading at a primary level and was able to do simple addition and subtraction. John was able to vocalize; however, due to dysarthria, speech was often unintelligible. Several mechanical and infrared switches were tested on his left finger but were shown to be unreliable and difficult to position due to involuntary movements of his head and arms. John had also experimented with an eyebrow switch, which was reported to be overly fatiguing, and a grip switch with which he had difficulty releasing. In addition, he tried a commercially available sound activated switch which interfered with his hearing aid, worked unreliably in noisy environments and was reportedly overly fatiguing. John was interested in using a switch for environmental control (e.g., changing TV channels/volume), playing games, as well as for educational purposes. His occupational therapist had worked with him for 3 years.

\subsubsection{Participant 3: Ethan}

Ethan was an 11.8 year old male. He was diagnosed with spastic quadriplegic cerebral palsy. He had good eyesight and wore bilateral hearing aids for medial loss of hearing in both ears. He used a wheelchair and had hypertonia in his arms and legs. He had good cognitive functioning and good memory. Ethan was able to vocalize, but due to dysarthria, his speech was often unintelligible. Several switches had been tried in the past, including mechanical switches mounted near his head, cheek, tongue, eyebrow, and feet, which were all reported to be overly fatiguing. Mounting of the mechanical switches also posed safety risks as Ethan had excessive involuntary movements. In addition, a sound activated switch had also been explored as an access modality, but it interfered with his hearing aid and was unreliable in the presence of environmental noise. Ethan's main interest for using a switch was for reading on a computer. His occupational therapist had worked with him for 3 years.

\subsubsection{Participant 4: Dorothy}

Dorothy was a 14.5 year old female. She was diagnosed with spastic quadriplegic cerebral palsy and had severe hypertonia in her arms and legs as well as spastic head movements. She used a wheelchair and her hearing and vision were within normal limits. Her cognitive function had not been assessed but she was reading at a primary level. Opportunities to develop her literacy skills had been limited by extended school absences secondary to pain, as well as the lack of a feasible switch access method. Dorothy was attentive and was able to understand speech as well as speak, but due to dysarthria, speech was often unintelligible. She had a history of using a button-style mechanical switch placed in several places, including near her feet and behind her elbow; such solutions, however, were difficult to mount to her wheelchair and her movements were unreliable. Dorothy had also tried a sip and puff switch which had to be constantly repositioned due to her spastic head movements. Dorothy was interested in using a switch to access the internet, play games, and send email. Her occupational therapist had been working with her for 10 years.

\subsection{Description of Vocal Cord Vibration Detection Switch}

Fig. (1) depicts a block diagram of a recently-developed vocal cord vibration switch and its use as an access solution. The switch consists of a sensor, a dual-axis accelerometer, which is placed on the neck near the vocal cords with a cotton neckband with two adjustable straps and a safety release clip. Accelerometer axes were aligned to the anteriorposterior and superior-inferior anatomical axes. The sensor was connected to a microcontroller box which was responsible for analyzing the input signals, more specifically, to detect highly periodic vibrations of the vocal cords. Due to properties of the voice production system, highly periodic vocal cord vibrations are produced when voiced sounds (e.g., vowels), hums, or some consonants (e.g. 'b', 'd', 'g') are vocalized. In contrast, less periodic or aperiodic vibrations are produced with coughs, swallows, 


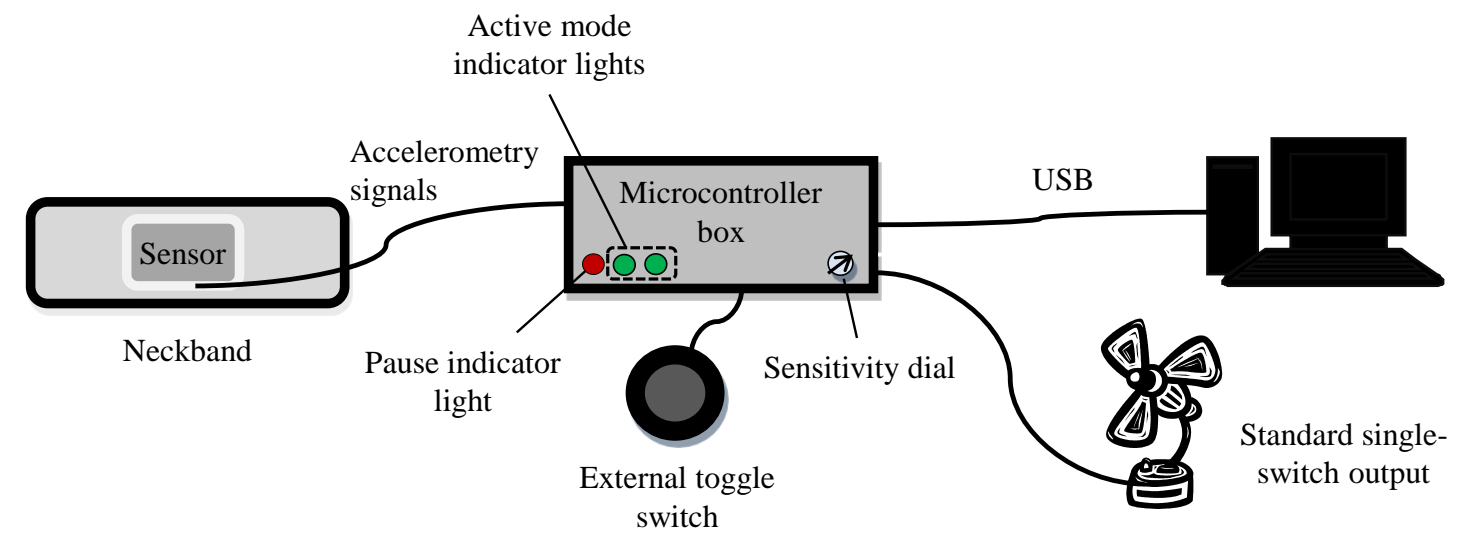

Fig. (1). Block diagram of the vocal cord vibration switch.

and throat clearing sounds [12]. By detecting only highly periodic vibrations, false switch activations due to usergenerated artifacts are avoided. Additionally, by harnessing vocal cord vibrations via an accelerometer, the switch becomes insensitive to environmental noise, thus overcoming the shortcomings of existing voice-based access methods. To account for individual differences in vocal fold function, the sensitivity, or level of periodicity to be detected, can be adjusted by the caregiver to suit the individual user.

A real-time implementation of the switch was developed in hardware using a PIC24FJ64GA004 microcontroller. The microcontroller box included the aforementioned adjustable knob as well as three indicator lights. A red light turned on to indicate that the system was in pause mode; a functionality made available to allow users to talk with communication partners (e.g., caregivers, teachers, parents) without causing erroneous switch activations. Pause mode could be activated by means of an external toggle switch as illustrated in the figure. Two green lights were indicative of detected vocalizations. The microcontroller was programmed to output a conventional switch output, a pre-specified keyboard stroke, or a mouse click. The device could connect to a computer via USB or connect to an external switchadapted device such as a fan. The microcontroller unit was designed such that it could be battery operated or powered via USB when connected to a computer. The system was portable and did not require special mounting. A more detailed description of the signal processing steps involved in periodicity detection is given elsewhere [12].

\subsection{Experimental Setup}

Upon enrolment in the study, participants and their caregivers were introduced to the device and received training on device setup and operation. Once participants were comfortable with using the switch, they were given the switch to take home and were encouraged to use it as often as desired. Participants were followed over a one month period after switch prescription. Within this study period, three switch assessments were conducted approximately 1-2 weeks apart. Each session was videotaped for subsequent analysis. Total session time was recorded and did not include breaks between activities. Sessions took place in a variety of settings such as the rehabilitation center, participants' schools, as well as their homes; all sessions had some degree of background noise. Participants were given at least three different activities, based on their personal interests, with which to use the vocal cord vibration switch; additional activities were given to those who reported being "bored" with only three activities. Experimental settings were customized to accommodate individual intellectual levels, endurance, and attention spans. Activities included computer e-book reading, cause-and-effect games on the computer, activation of switch-adapted devices (e.g., fan and pillow), as well as scanning activities (e.g., matching letters and prepositions). A description of the settings and activities for each participant can be found in Table $\mathbf{1}$.

\subsection{Switch Evaluation}

\subsubsection{Qualitative Measures}

Parents of all participants were given a demographic and a post-evaluation questionnaire. Therapists, in turn, were given a pre-evaluation questionnaire with questions related to previous switch history, physical limitations, and stimuli preferences, as well as a post-evaluation questionnaire. Participants were asked to rate their level of fatigue before and after the session based on the modified five point Borg scale $(1=$ Not tired; $2=\mathrm{A}$ little tired; $3=$ Moderately tired; $4=\mathrm{A}$ lot tired; and 5=Very tired). Upon the conclusion of the three sessions, participants were given a post participation survey. For the participants, the questionnaire consisted of yes/no questions as some participants were either too young, or did not have the cognitive capacity to answer questions on a 5 point Likert scale. Participants were asked the following six questions: 1) Do you like using the device? 2) Do you want to use it in the future? 3) Was it easy to use? 4) Did it make you tired? 5) Did it make your throat hurt? 6) Were you frustrated or mad when you used this device?

Therapists and caregivers, on the other hand, were asked to respond to the three statements using a 5-point Likert scale from $(1=$ strongly disagree to $5=$ strongly agree $)$. The statements were: 1) This switch was easy to set up compared with other switches, 2) This switch was reliable (it turns on when it is supposed to) for my client/child, 3) This switch was efficient (easy to use) for my client/child. Additionally, therapists and clients were asked open-ended questions such as 1) What are some of the perceived benefits and drawbacks of using the switch? and 2) What are suggestions for improvement? Caregivers, in turn, were given an additional 
Table 1. Experimental Settings for Each of the Four Participants

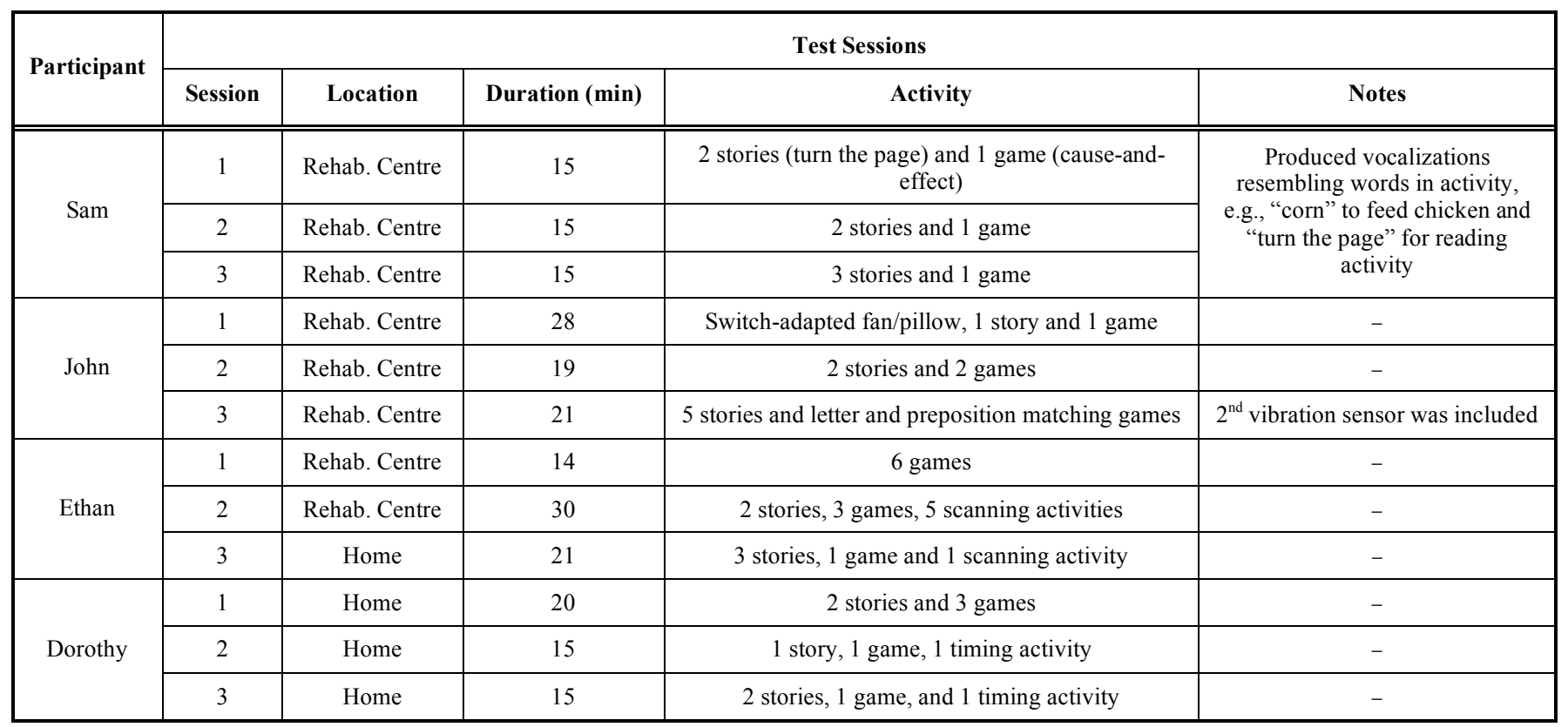

question relating to the amount of time the child used the switch at home during the one month period.

\subsubsection{Quantitative Performance Metrics}

In this study, switch sensitivity and specificity were used as performance metrics and were calculated per session. Sensitivity and specificity are given by:

Sensitivity $=\frac{\text { Number of True Activations }}{\text { Number of True Activations }+ \text { Number of False Negatives }} \times 100 \%$,
Specificity $=\frac{\text { Number of True Activations }}{\text { Number of True Activations }+ \text { Number of False Positives }} \times 100 \%$,

where a true activation indicated that the device was correctly activated upon the participant vocalizing, a false activation indicated that the device was erroneously activated without a user-generated vocalization, and a false negative indicated that despite the user vocalizing, the switch did not activate. Additionally, per-session perceived exertion level was calculated as the difference in perceived tiredness score between the end and the beginning of each session; larger exertion levels indicate increased fatigue.

\section{RESULTS}

\subsection{Quantitative Performance}

Sam reported no change in perceived exertion levels for all three sessions. During the first session, he had an initial and final perceived tiredness level of 3 (moderately tired). During the second and third sessions, his reported levels were 2 (a little tired) and 1 (not tired), respectively. Fig. (2a) illustrates the sensitivity and specificity levels obtained for each of the three sessions. For the first session, 59\% sensitivity and $100 \%$ specificity were observed. The low sensitivity was likely due to Sam's fatigue (self report) which caused his voice to be very breathy and vocalizations to sound whispered, thus not generating many periodic vocal cord vibrations. Sam's performance improved for the subsequent sessions and an average sensitivity of $73.3 \%$ and an average specificity of $98.1 \%$ were observed. Due to his small neck circumference, the neckband had to be readjusted once and the sensitivity dial on the processing unit had to be readjusted for his first and third sessions to allow for his whispered speech to be detected. A drawback of decreasing the sensitivity of the switch was that user-generated artifacts with lower periodicity were erroneously detected, hence decreasing switch specificity, as seen for session 3 in Fig. (2a).

Like Sam, John reported no change in perceived exertion levels for all three sessions. At the start and finish of the first session he reported his fatigue level to be 3 (moderately tired). For the second and third sessions, he reported his fatigue to be at level 1 (not tired). He usually vocalized by saying "ah". Due to spastic head movement, his neckband was adjusted at least once each session such that the sensor was positioned around the vocal cord. During his third session, two sensors were used to alleviate the problems associated with the neckband moving during John's numerous involuntary head movements. During this last session, however, a faulty cable was detected and errors were not included in the performance depicted by Fig. (2b). The errors, however, were frustrating for John and his overall performance on the third session may have been affected. Overall, the switch had average sensitivity of $80.0 \%$ and an average specificity of $98.8 \%$.

Ethan also reported no change in perceived exertion levels for all three sessions. At the beginning and end of the first session he reported a fatigue level of 1 (not tired). At the beginning and end of sessions 2 and 3, he reported a fatigue level of 2 (a little tired). He usually vocalized by saying "go". Ethan would often complain about the position of his legs and ask his caregivers to adjust his legs during the middle of the sessions. False switch activations resultant from such vocalizations were not included in the performance measures depicted by Fig. (2c). His neckband had to be readjusted at least once each session to account for neckband displacement from spastic head movements. An 


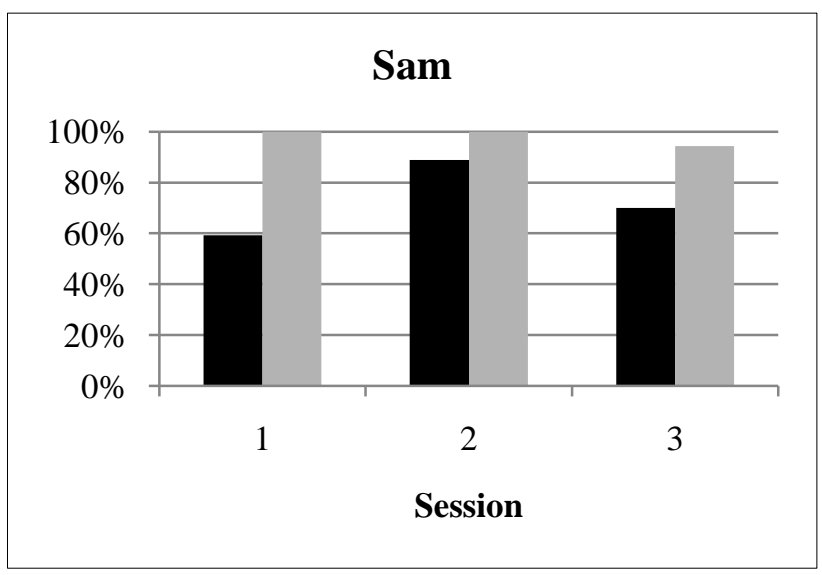

a)

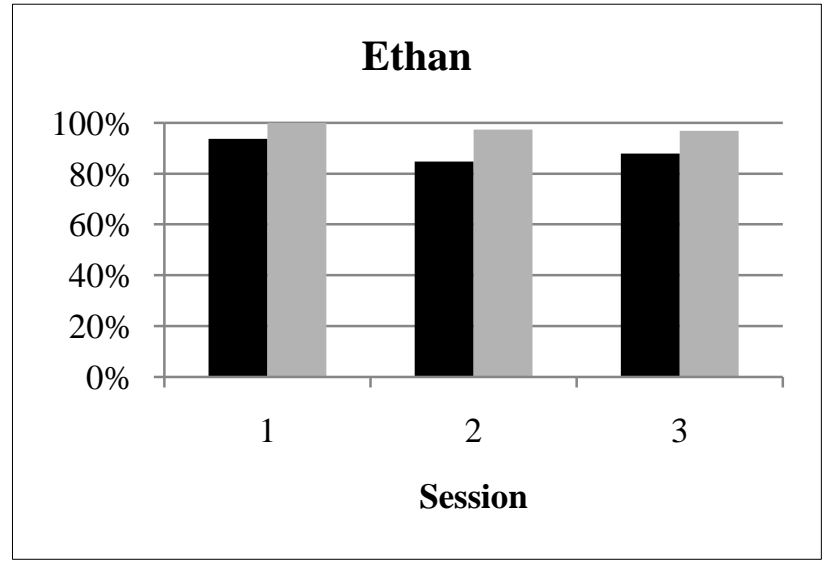

c)

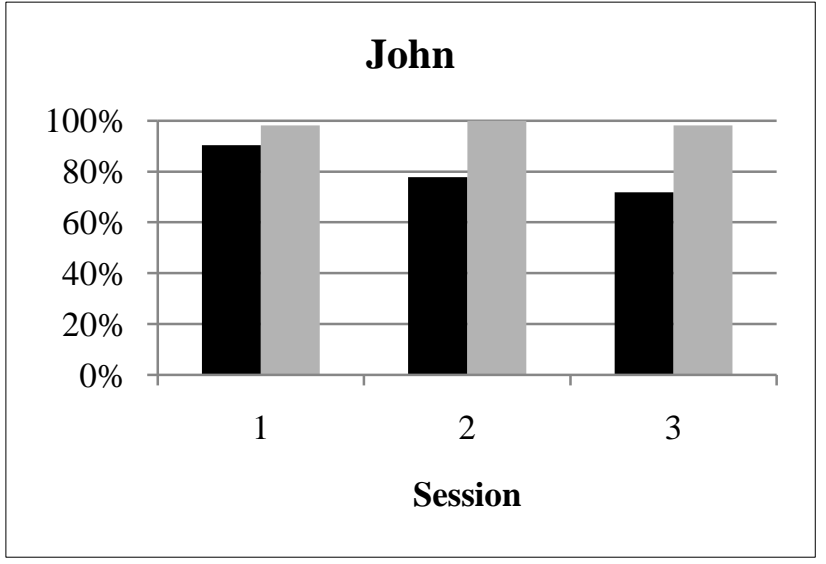

b)

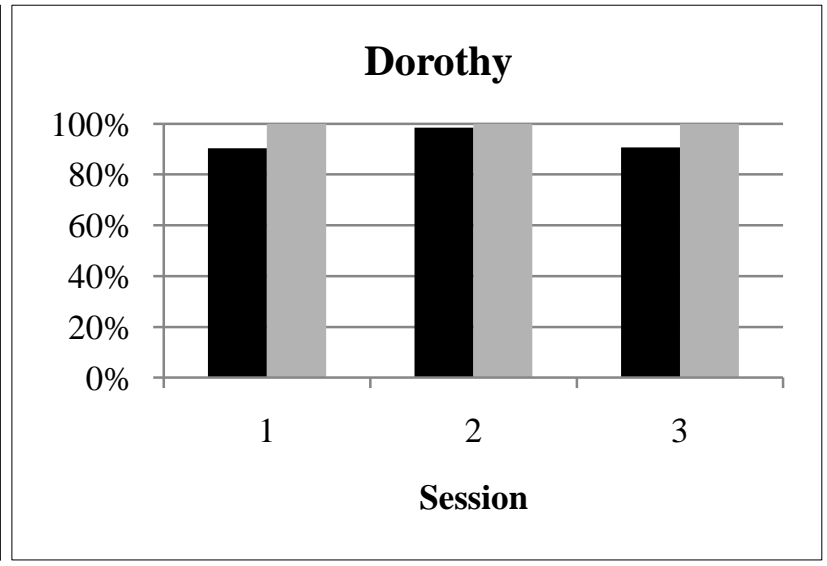

d)

Fig. (2). Sensitivity (black bars) and specificity (gray bars) of the vocal cord vibration switch for a) Sam, b) John, c) Ethan, and d) Dorothy.

average sensitivity of $88.8 \%$ and an average specificity of 98.1\% were obtained.

Dorothy did not experience a change in perceived tiredness levels for the first two sessions (both began and ended at level 1 - not tired) and experienced a 1-point change during her third session (began at 1 and ended at $2-$ a little tired). Such change, however, may have been due to sleep deprivation, as her father reported that she did not sleep well the night before the third session. Dorothy's method of activating the switch was by humming, i.e., producing the sound "hmm". Her sessions at home were performed in the presence of background noise from the radio and people talking. Dorothy's performance was fairly constant throughout the three sessions, as shown in Fig. (2d). The average sensitivity of her device over the three sessions was $93.2 \%$ and average specificity was $100 \%$.

\subsection{Survey Results}

Table 2 reports the results of the post-evaluation questionnaire given to the caregiver and clinician. As observed, all caregivers and clinicians either agreed or strongly agreed that the vocal cord vibration switch was easy to set up. Three of the four caregivers agreed that the switch worked reliably/effectively and one strongly agreed. Most participants were able to use the switch at least once a week during the month-long study. Table 3 reports the results of the post-evaluation questionnaire given to the participants. As observed, all participants indicated that they liked the device, found it easy to use, did not feel frustrated when using it, and wanted to continue using it. One participant (Ethan) felt the device was a little tiring to use and indicated feeling pain in his throat when using the device.

\section{DISCUSSION}

\subsection{Harnessing Vocal-Fold Vibrations for Assistive Technology Development}

This study investigated the viability of a recentlydeveloped periodic throat vibration detection switch for individuals with complex communication needs who are able to vocalize. Key advantages of the device include its insensitivity to environmental noise and minimal physical demand on the user. Most participants experienced no change in perceived fatigue levels from the beginning to the end of each session. At most, a 1-point change was observed. Overall, the vocal cord vibration switch was able to remove user-generated unintended throat sounds (e.g., coughs) with an average specificity ranging from $98.1 \%$ to $100 \%$ for the four participants. Such results indicate that the device would 
Table 2. Caregiver and Clinician Post-Participation Survey Results Based on a 5-Point Liker t Scale (1=Strongly Disagree, 2=Disagree, $3=$ Undecided, 4=Agree, 5=Strongly Agree)

\begin{tabular}{|c|c|c|c|c|c|c|c|}
\hline \multirow{2}{*}{ Participant } & \multicolumn{2}{|c|}{ Ease of Set Up } & \multicolumn{2}{c|}{ Worked Reliably } & \multicolumn{2}{c|}{ Worked Efficiently } & \multirow{2}{*}{ Use of Switch During Study } \\
\cline { 2 - 8 } & Caregiver & Clinician & Caregiver & Clinician & Caregiver & Clinician & $4-5 \mathrm{x} / \mathrm{week}$ \\
\hline \hline Sam & 4 & 5 & 4 & 4 & 4 & 4 & $1 \mathrm{x} / \mathrm{week}$ \\
\hline John & 5 & 4 & 4 & 4 & 4 & 4 & $2-3 \mathrm{x} / \mathrm{week}$ \\
\hline Ethan & 5 & 4 & 5 & 4 & 5 & 4 & $1-3 \mathrm{x} / \mathrm{week}$ \\
\hline Dorothy & 4 & 5 & 4 & 5 & 4 & 5 & \\
\hline
\end{tabular}

Table 3. Participant Post-Participation Survey Results Based on Yes/No Questions

\begin{tabular}{|c|c|c|c|c|c|c|}
\hline Participant & Like the Device & Future Use & Ease of Use & Device Tiring & Throat Pain & Frustration \\
\hline \hline Sam & Yes & Yes & Yes & No & No & No \\
\hline John & Yes & Yes & Yes & No & No & A little \\
\hline Ethan & Yes & Yes & Yes & A little & No & No \\
\hline Dorothy & Yes & Yes & Yes & No & No \\
\hline
\end{tabular}

be a good fit for those who need to be cued to speak (i.e., partner's speech would not cause erroneous switch activations), or would like to use the device in noisy environments such as at home or at school.

The vocal cord vibration switch was able to pick up most vocalizations; however the sensitivity depended on the participant. Some participants were able to vocalize more readily than others and some participants had very breathy and whispered vocalizations which did not generate highly periodic vocal cord vibrations. As a consequence, this access pathway may be easier to control for individuals who are able to produce voiced sounds. Nonetheless, it may still be a viable pathway for those who have breathy vocalizations with the assistance of breath control and/or voice training, as previous studies have suggested that voluntary vocalizations can be developed in some individuals with multiple and severe disabilities $[9,13]$.

\subsection{Challenges and Benefits}

A factor that we observed affected switch performance was head positioning. It was observed that if the neckband was not sufficiently tight, the sensor would not pick up vocal cord vibrations when the head was leaned forward and down. For participants who had varied voice control, or who had raspy breathing, the switch's sensitivity had to be adjusted accordingly. In addition, since the vocal cord vibration switch detected any periodic vibration, those who used speech to communicate with partners would need an alternate method to disable switch activation. For individuals with a secondary access site, this could be achieved by activating a second switch to toggle the vibration switch to pause mode. An alternate option for individuals without a reliable secondary site is to incorporate vocalization duration into switch decisions. As an example, long-duration speech segments (e.g., greater than one second) can be filtered and switch activations would only occur with short-duration vocalizations in the order of tens of milliseconds.
In terms of mobility, once the neckband was in place, mounting was not required, thus participants could use the switch in numerous places and/or positions. However, the design of the neckband presented some challenges during this study. As examples, involuntary head movements shifted the sensor away from the vocal cords and targeted vibrations were not measured. Similarly, excessive contractions of the neck muscles would make the neckband loose, thus also precluding the detection of targeted vibrations. In addition, many participants did not like the safety release feature as it disengaged too easily. For those with excessive head movements, the neck began to chafe from the neckband. Improvements in neckband design will likely lead to improvements in overall switch performance.

Further improvements suggested by the participants and their caregivers/clinicians included making the switch wireless and extending the number of switch outputs to two or more. The latter is currently under development and is based on discriminating between different vocal cord vibration frequencies (high or low pitch) and vocalization durations (short or long).

Overall, the vocal cord vibration switch was deemed to be a viable solution for all four participants who, since the conclusion of this study, have been using it for entertainment and classroom activities. Unfortunately, participants did not possess a reliable secondary access method for comparison purposes. Nonetheless, as testament of the benefits attained with the switch, Dorothy's clinician remarked that in the last 10 years, she had never seen Dorothy use the computer for such an extended period of time. Ethan's caregiver wrote that, "It's the most reliable switch we have tried." In all, the benefits of using the switch seemed to outweigh the drawbacks for all four participants involved in this study.

\subsection{Study Limitations}

The small sample size of this descriptive study makes it difficult to extrapolate results and to infer about the usability 
of the device with other user populations. Larger controlled studies are still needed to determine the generality of the findings. Moreover, as mentioned in Section 3.1, the use of a single sensor on the neckband may not be optimal for individuals with excessive spastic head movement as sensors may become dislodged from the vocal-fold region of the neck. Future studies will need to investigate the gains obtained with using an array of sensors placed around the neck. The current version of the system limits individuals to single-switch activities. Future studies should aim at expanding to multiple outputs to allow for multiple-switch usage. Possible avenues to achieve this goal might be to harness vocalizations of different durations (e.g., long/short) or of different pitch levels (low/medium/high).

\section{CONCLUSION}

This study evaluated the viability of a recently-developed vocal cord vibration switch for four children with severe and multiple disabilities over a period of one month. Across participants, the device had an average sensitivity and specificity of $83.8 \%$ and $98.8 \%$, respectively. The switch was easy to set up, non-fatiguing, robust against usergenerated noises, and insensitive to environmental noise. Despite issues with neckband design, participants, their caregivers, and clinicians unanimously agreed that the device was an acceptable access method for communication, entertainment, and environmental control purposes.

\section{ACKNOWLEDGEMENTS}

We would like to thank Ka Lun Tam and Pierre Duez for their invaluable technical support in developing the vocal cord vibration switch. We would also like to thank Vince Gracco, Anne Marie Renzoni, Cynthia Tam, Susie Rothman, Amanda Baumann, Marlene Ferriggi, and Laura Franks for their participation and insight. This research was funded by the WB Foundation and IBM Canada.

\section{REFERENCES}

[1] Huer MB, Lloyd, LL. AAC users' perspectives on augmentative and alternative communication. Augment Altern Commun 1990; 6(4): 242-9.

[2] Beukelman D, Mirenda P. Augmentative and alternative communication: supporting children and adults with complex communication needs. Paul H: Brookes Publishers 2005.

[3] Lloyd LL, Fuller DR, Arvidson H. Introduction and overview. In Lloyd LL, Fuller DR, Arvidson H, Eds. Augmentative and alternative communication: a handbook of principles and practices. Needham Heights, MA: Allyn \& Bacon 1997, pp.1-17.

[4] Minister of Industry Statistics Canada. Social and Aboriginal Statistics Division. Participation and activity limitation survey 2006: analytical report. Ottawa, ON 2007.

[5] McNaughton D, Nelson B, Nelson D. AAC technologies to enhance participation and access to meaningful societal roles for adolescents and adults with developmental disabilities who require AAC. Augment Altern Commun 2007; 23(3): 217-29.

[6] Hoppestad BS. Inadequacies in computer access using assistive technology devices in profoundly disabled individuals: an overview of the current literature. Disabil Rehabil Assist Technol 2007; 2(4): 189-99.

[7] Man DWK, Wong MSL. Evaluation of computer-access solutions for students with quadriplegic athetoid cerebral palsy. Am J Occup Ther 2007; 61: 355-64.

[8] Noyes JM, Frankish CR. Speech recognition technology for individuals with disabilities. Augment Altern Commun 1992; 8(4): 297-303.

[9] Lancioni GE, Lems S. Using a microswitch for vocalization responses with persons with multiple disabilities. Disabil Rehabil 2001; 23: 745-8.

[10] Lancioni GE, Singh NN, O'Reilly MF, et al. A voice-detecting sensor and a scanning keyboard emulator to support word writing by two boys with extensive motor disabilities. Res Dev Disabil 30: 203-9.

[11] Lancioni, GE, Singh N, O'Reilly M, Oliva D, Groeneweg J. Enabling a girl with multiple disabilities to control her favourite stimuli through vocalization and a dual-microphone microswitch. J Vis Impair Blind 2005; 99(3): 133-40.

[12] Chan J, Falk TH, Teachman G, Morin-McKee J, Chau T. Evaluation of a non-invasive vocal cord vibration switch as an alternative access pathway for an individual with hypotonic cerebral palsy - a case study. Disabil Rehabil Assist Technol 2010; 5(1): 69-78.

[13] Yorkston K, Beukelman D, Strand E, Bell K. Management of motor speech disorders in children and adults. Austin, TX: Pro-Ed Publishers 1999. 